\title{
An early increase in the disialoganglioside GD3 contributes to the development of neuronal apoptosis in culture
}

\author{
D Melchiorri ${ }^{1,3}$, F Martini $^{2}$, E Lococo ${ }^{2}$, R Gradini $^{2}$, E Barletta $^{1}$, \\ R De Maria ${ }^{4}$, A Caricasole ${ }^{1,3}$, F Nicoletti ${ }^{\star, 1,3}$ and L Lenti ${ }^{2}$ \\ 1 Department of Human Physiology and Pharmacology, University of Rome \\ 'La Sapienza', Italy \\ 2 Department Experimental Medicine and Pathology, University of Rome \\ 'La Sapienza', Italy \\ ${ }^{3}$ I.N.M. Neuromed, Pozzilli, Italy \\ ${ }^{4}$ Laboratory of Hematology and Oncology, Istituto Superiore di Sanità, Rome, \\ Italy \\ ${ }^{*}$ Corresponding author: F Nicoletti, Department of Human Physiology and \\ Pharmacology, The University of Rome 'La Sapienza', Rome Italy. \\ Tel: +39-06-49912969; E-mail: ferdinandonicoletti@hotmail.com
}

Received 11.9.01; revised 5.12.01; accepted 9.1.02 Edited by F Miller

\begin{abstract}
We induced apoptosis in primary cultures of cerebellar granule neurons by switching the growing medium into a medium containing lower concentrations of $\mathrm{K}^{+}(5$ or $10 \mathrm{mM}$ instead of $25 \mathrm{mM}$ ) or, alternatively, by addition of staurosporine. The apoptotic phenotype was always preceded by an early increase in the intracellular levels of the disialoganglioside GD3, which peaked at 2-6 $\mathrm{h}$ and returned back to normal at $12 \mathrm{~h}$. GD3 synthase, the enzyme that forms GD3 from the monosialoganglioside GM3, was also induced at early times after the induction of apoptosis in granule cells. Immunofluorescent staining showed that GD3 increased in neuronal cell bodies and neurites, but was never localized in cell nuclei. In cultures switched into a low $\mathrm{K}^{+}$-containing medium, exogenously applied GD3, but not the disialoganglioside GD1a, accelerated the development of neuronal apoptosis. In contrast, the antisense-induced knock-down of GD3 synthase was protective against granule cell death induced by lowering extracellular $\mathrm{K}^{+}$from 25 to 10 - but not 5 - mM. These results demonstrate that an early and transient increase in GD3 synthesis is one of the factors that contribute to the induction of neuronal apoptosis in culture.

Cell Death and Differentiation (2002) 9, 609-615. DOI: 10.1038/ sj/cdd/4401020
\end{abstract}

Keywords: GD3; cerebellar granule neurons; apoptosis; neuronal cultures

Abbreviations: DIV, days in vitro; NF- $\kappa$ B, nuclear factor- $\kappa$ B; s.C., serum containing; s.f., serum-free; HPTLC, high performance thin layer chromatography; PBS, phosphate buffered saline

\section{Introduction}

Neuronal apoptosis is widely implicated in a variety of physiological and pathological processes, such as the elimination of supranumerary neurons during CNS development or the progressive loss or vulnerable neurons in acute or chronic neurodegenerative disorders. ${ }^{1-3}$ In spite of an extensive research, the molecular events that mediate the early steps of the apoptotic signal in neurons are largely unknown. In myeloid and lymphoid tumour cells, an early and transient increase in the formation of the disialoganglioside GD3 is required for the development of apoptosis in response to Fas/CD95 receptor activation. ${ }^{4}$ A role for GD3 in apoptosis is strengthened by the evidence that GD3 induces mitochondrial permeability transition with ensuing release of Cytochrome $c$ and caspase activation. ${ }^{4-9}$ Alternatively, GD3 has been shown to enhance apoptosis by preventing the nuclear translocation of nuclear factor- $\kappa \mathrm{B}(\mathrm{NF}-\kappa \mathrm{B})$, thus suppressing the NF- $\kappa \mathrm{B}$-dependent survival pathway. ${ }^{10}$ Although gangliosides are major constituents of the vertebrate brain, the role of GD3 in neuronal apoptosis is still unexplored. We have addressed this issue in cultured cerebellar granule cells, which offer the advantage of a high homogeneity of the neuronal population. Cultured granule cells are usually grown under chronic depolarizing conditions (i.e. in medium containing $25 \mathrm{mM} \mathrm{K}^{+}$), which are required for cell maturation and survival. In these cultures, apoptosis may be induced by switching the growing medium into a medium containig lower concentrations of $\mathrm{K}^{+}$, independently of the presence of serum. This particular form of 'apoptosis by trophic deprivation' incorporates features of the developmental apoptosis of granule cells that physiologically occurs during early postnatal life, and has largely been used for the study of the molecular determinants of neuronal death. ${ }^{11-16}$ We report that the induction of apoptosis in cultured granule cells is associated with an early and transient increase in the intracellular levels of GD3, and that GD3 depletion is protective against neuronal death.

\section{Results}

Cultured cerebellar granule cells grown in medium containing $10 \%$ FCS and $25 \mathrm{mM} \mathrm{K}^{+}$(K25) were viable and fully differentiated after 6 days in vitro (DIV). For the induction of apoptosis, we have switched the growing medium either into a fresh serum-free (s.f.) medium containing $5 \mathrm{mM} \mathrm{K}^{+}(\mathrm{K} 5)$ or into a conditioned medium collected from sister cultures grown in the presence of serum (serum containing or s.c.) but with $10 \mathrm{mM} \mathrm{K}^{+}$(K10). We did not switch K25 cultures into fresh scK10 medium to avoid the induction of excitotoxic neuronal death by the micromolar amounts of glutamate present in fresh serum. ${ }^{17}$ Cultured granule cells switched from K25 into sfK5 or scK10 developed substantial apoptotic death within 48-72 h (Figure 1). No phenotypic hallmarks of 
apoptosis (chromatin fragmentation or nuclear picnosis) were visible by Hoechst staining during the first $12 \mathrm{~h}$ following the medium switch (Figure 1). 'Low- $\mathrm{K}^{+\prime}$-induced apoptosis was associated with mitochondrial membrane depolarization, as indicated by the increase in the green fluorescence of the dye JC-1 (Figure 2). Apoptosis was also induced by applying the protein kinase inhibitor, staurosporine $(50 \mathrm{nM})$, to $\mathrm{K} 25$

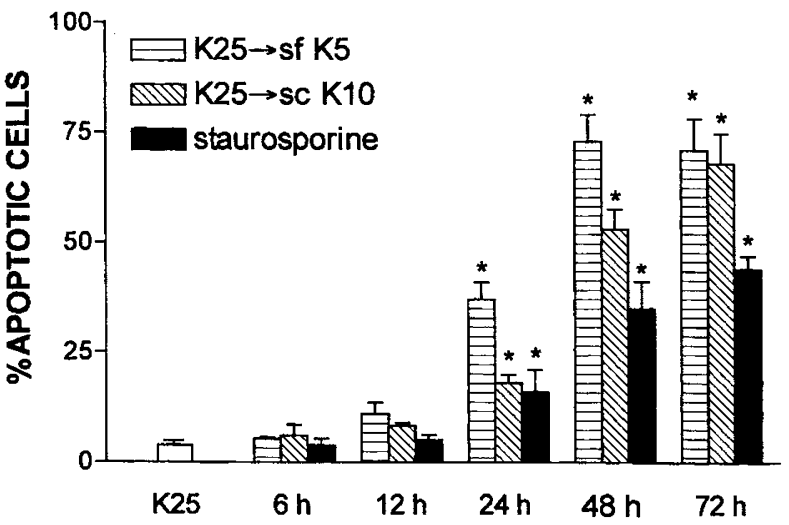

Figure 1 Development of apoptosis in cultured cerebellar granule cells treated as follows: (i) switch of the growing medium (K25) into a fresh K5 medium lacking foetal calf serum (sfK5); (ii) switch of the growing medium into a medium collected from sister cultures grown in $\mathrm{K} 10$ in the presence of serum (scK10); (iii) addition of $50 \mathrm{nM}$ staurosporine to the growing medium. No increase in the percentage of apoptotic neurones was seen in cultures switched from K25 into K25 at any time (not shown). Apoptotic death was assessed by Hoechst staining. Values are means + S.E.M. of 5-6 determinations. ${ }^{*} P<0.05$ (One-way ANOVA *Fisher's PLSD) as compared to the respective values at 6 and $12 \mathrm{~h}$ cultures. At these concentrations, staurosporine produced apoptotic death in about $35 \%$ of neurons after $72 \mathrm{~h}$ (Figure 1). In all these models of trophic deprivation, the phenotypic hallmarks of apoptosis were preceded by a transient increase in the intracellular levels of GD3, as detected by HPTLC combined with immunodetection. The increase in GD3 peaked between 4 and $6 \mathrm{~h}$ after switching the culture medium from K25 into sfK5 or scK10, whereas it was maximal $2 \mathrm{~h}$ after the addition of staurosporine. GD3 levels returned back to normal after $12 \mathrm{~h}$ (Figure 3). HPTLC analysis performed in cultures switched from K25 into scK10 also showed an early increase in the levels of GD1b and GT1b, which are generated from GD3 metabolism (not shown). Immunofluorescence analysis carried out at different times after switching the medium from K25 into sfK5 (Figure 4) or scK10 (not shown) showed that the increase in GD3 (green in Figure 4) was localized both in the cell bodies and neurites of granule cells. The absence of co-localization between GD3 immunofluorescence and propidium iodide (red in Figure 4) indicated that GD3 accumulated outside the cell nucleus. GD3 was nearly undetectable in K25 control cultures (Figure 4). Apoptosis was also associated with an early induction of the enzyme GD3 synthase, which was detected at 2 and $4 \mathrm{~h}$ after switching the medium (Figure 5).

To examine whether GD3 had any role in the induction of apoptosis, we firstly applied exogenous GD3 to the cultures. Addition of GD3 (up to $100 \mu \mathrm{M}$ ) to K25 cultures had no effect on granule cells viability. However, when applied to the cultures immediately after switching the medium from K25 into scK10, GD3 $(10 \mu \mathrm{M})$ accelerated the development of apoptosis in granule cells. In the presence of GD3, an increase in the percentage of

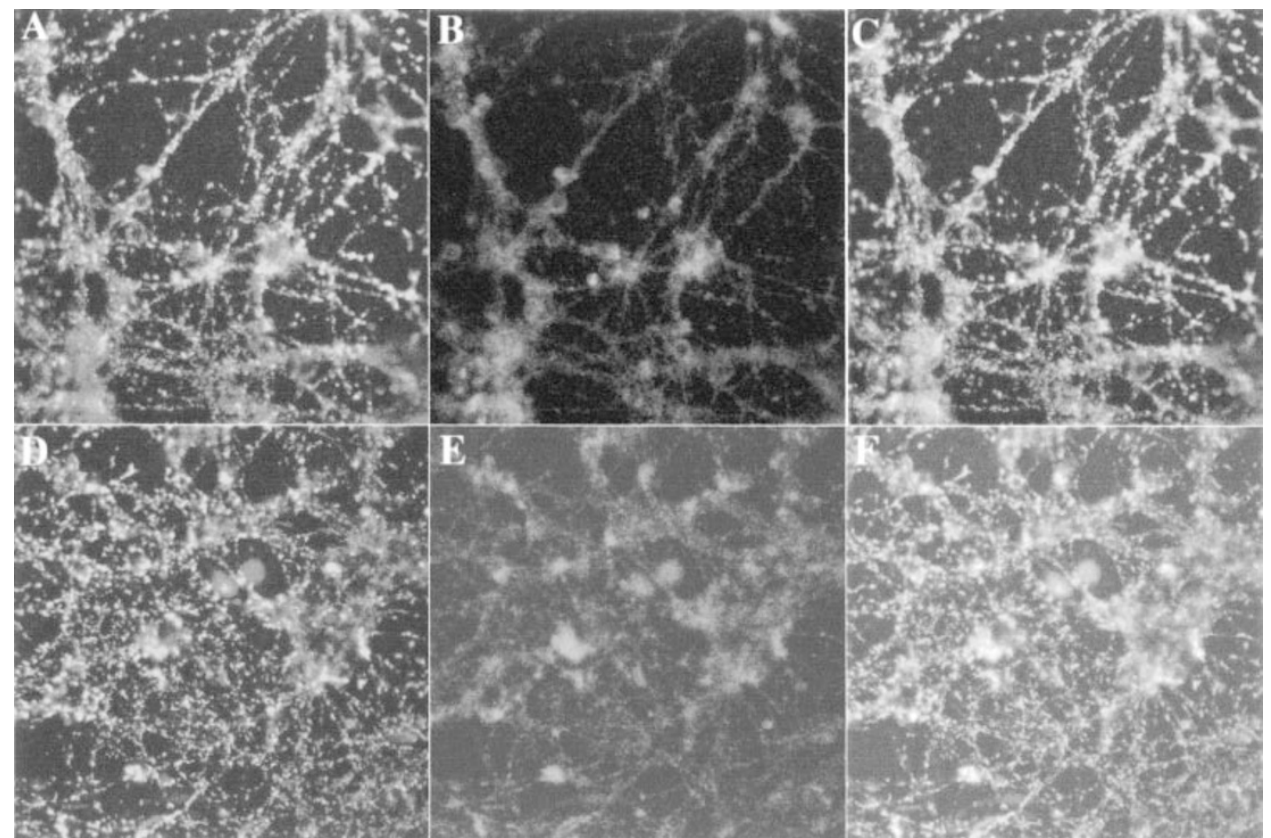

Figure 2 Changes in mitochondrial membrane potential $12 \mathrm{~h}$ after switching the medium of cultured granule cells from K25 into scK10. Cultures were stained with the mitochondrial dye, JC-1, which emits red fluorescence in its aggregate form with high mitochondrial membrane potentials and green fluorescence in its monomeric form with low mitochondrial membrane potentials. Fluorescence in control cultures (switched from K25 into K25) and in cultures switched from K25 into scK10 are shown in $(\mathbf{A})$ and $(\mathbf{B})$, and $(\mathbf{D})$ and $(\mathbf{E})$, respectively. Co-localization is shown in $(\mathbf{C})$ and $(\mathbf{F})$, respectively 
A
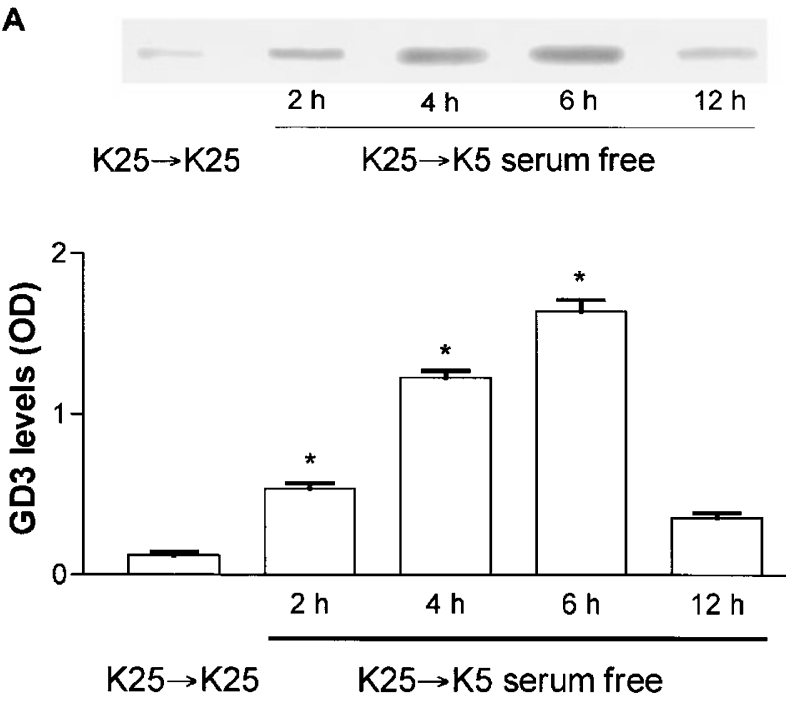

B

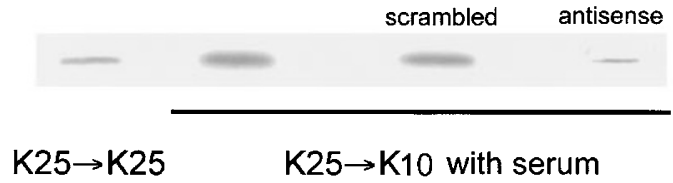

C

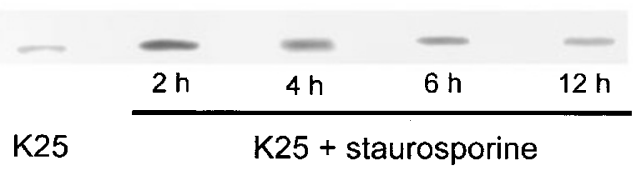

Figure 3 (A) Early increase in GD3 levels in cultured granule cells after switching the K25 medium into a K5 medium lacking foetal calf serum. Densitometric values are also shown $\left(n=3-4 ;{ }^{*} P<0.05\right.$ vs control values; One-way ANOVA+Fisher's PLSD). Cell number was approximately the same $\left(2 \times 10^{6} /\right.$ dish $)$ in all dishes used for HPTLC. Values obtained $4 \mathrm{~h}$ after switching the K25 medium in K25 medium collected from sister cultures are shown in the first lane and considered as controls. These 'control' values did not differ from those obtained from K25 cultures that did not undergo the medium switch (compare the first lane of $(\mathbf{A})$ and $(\mathbf{C})$ ). There was no change in cell viability and cell number up to $12 \mathrm{~h}$ after switching the medium from K25 into sfK5. (B) Representative HPTLC of GD3 in cultured cerebellar granule cells, $4 \mathrm{~h}$ after switching the K25 medium into a scK10 medium collected from sister cultures. The effect of a 2-day treatment with a GD3 antisense or a scrambled oligonucleotides (both at $2 \mu \mathrm{M}$ ) is also shown. Cultures switched from K25 into K25 (4 h) are shown as controls. (C) Time-dependent increase in GD3 levels in K25 cultures treated with $50 \mathrm{nM}$ staurosporine. Experiments in (B) and (C) were repeated two times with similar results

apoptotic neurons was detected after 4, 24 and $48 \mathrm{~h}$ after the medium switch. No increase in granule cell apoptosis was induced by GD1a, a disialoganglioside that belongs to the 'a' series (Figure 6). We also examined the role of endogenous GD3 by treating the cultures with endcapped antisense oligonucleotides directed against GD3 synthase, the enzyme that converts GM3 into GD3. K25 Cultures were treated once daily for 2 days with antisenses $(2 \mu \mathrm{M})$ or with a scrambled oligonucleotide
$(2 \mu \mathrm{M})$, and then were switched into scK10 for the assessment of GD3 levels (after $4 \mathrm{~h}$ ) or apoptotic death (after $48 \mathrm{~h}$ ). In cultures tested for apoptotic death, antisense or scrambled oligonucleotides were applied once more immediately after the medium switch. Antisense treatment completely abolished the early increase in GD3 levels (Figure 3B), and significantly reduced the extent of apoptotic neuronal death in cultures switched from K25 into scK10 (Figure 7). GD3 antisenses were not protective when a more substantial apoptosis was induced by switching the medium from K25 into sfK5 (not shown).

\section{Discussion}

GD3, which is synthesized from GM3 by the enzyme $\alpha-2,8$ sialyltransferase (GD3 synthase), is the metabolic precursor of gangliosides of the 'b' series (such as GD1b, GT1b and GQ1b). GD3 is heavily expressed in the embryonic nervous system and, particularly, in rapidly proliferating neuroprogenitor cells. GD3 levels decline substantially at late stages of CNS development and are low in post mitotic-neurones. ${ }^{18-21}$ Interestingly, GD3 increases in the brain of patients with Alzheimer's disease, ${ }^{22}$ Creutzfeld-Jakob disease, and subacute sclerosis panencephalitis, ${ }^{23}$ although the significance of this increase is obscure. GD3 levels seem to correlate with cell proliferation, as suggested by the evidence that overexpression of GD3 synthase in PC12 cell enhances the proliferation rate via a sustained activation of TrkA and ERK1/ $2,{ }^{24}$ and that a decreased proliferation is observed in melanoma AbC1 cells depleted of GD3. ${ }^{25}$ However, an enhanced GD3 formation can also induce neuritic growth and cholinergic differentiation in Neuro2a cells. ${ }^{26}$ In our experiments, cultured cerebellar granule cells grown in K25 at 6 DIV, which were viable and fully differentiated, had low levels of both GD3 and GD3 synthase. In these cultures, GD3 was mainly localized in the few astrocytes present as contaminants (not shown; see refs. ${ }^{27,28}$ ). Interestingly, GD3 expression in granule cells was markedly increased a few hours after the induction of apoptosis. Apoptosis was induced by switching the medium from K25 into sfK5 or scK10, or, alternatively, by applying the broad protein kinase inhibitor, staurosporine. In all models, the increase in GD3 peaked 2$4 \mathrm{~h}$ after the induction of apoptosis, i.e. many hours before the appearance of the apoptotic phenotype. Therefore, an increase in GD3 formation represents an early event in the development of neuronal apoptosis. GD3 appeared to be localized in the cell body and neurites and was never detected in the nuclei of granule cells committed to death. GD3 may contribute to the apoptotic program by disrupting the mitochondrial transmembrane potential leading to the release of Cytochrome $c$ and activation of caspase 9, as shown in other cellular models. ${ }^{4-6}$ The change in mitochondrial membrane potential observed in granule cells undergoing apoptosis is consistent with this hypothesis. The levels of GD3 synthase, the enzyme that forms GD3 from GM3, increased 2 and $4 \mathrm{~h}$ after switching the cultures from the K25 medium into sfK5, suggesting a de novo synthesis of GD3. This provide one of the few demonstrations of an induction of GD3 synthase in cells committed to death. To demonstrate 


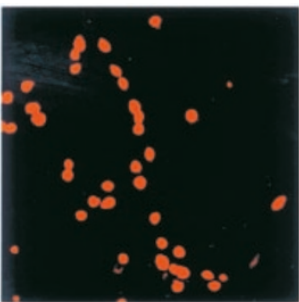

$\mathrm{K} 25 \rightarrow \mathrm{K} 25$

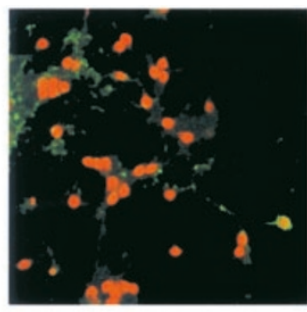

0,5

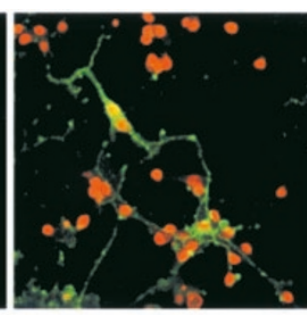

2

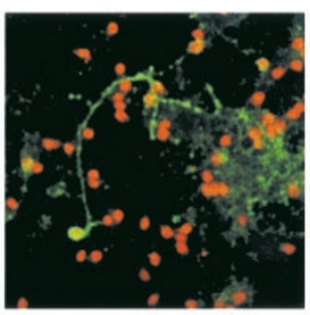

4

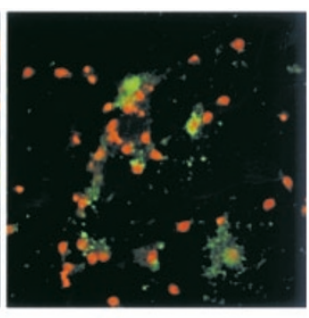

$12 \mathrm{~h}$

Figure 4 Immunofluorescent analysis of GD3 (green) $0.5-12 \mathrm{~h}$ after switching the K25 medium into a sfK5 medium. Note the lack of co-localization between GD3 and propidium iodide (red). Control cultures ( $12 \mathrm{~h}$ after switching the medium from K25 into sfK25) are also shown. A similar pattern of GD3 expression was observed in cultures switched from K25 into scK10

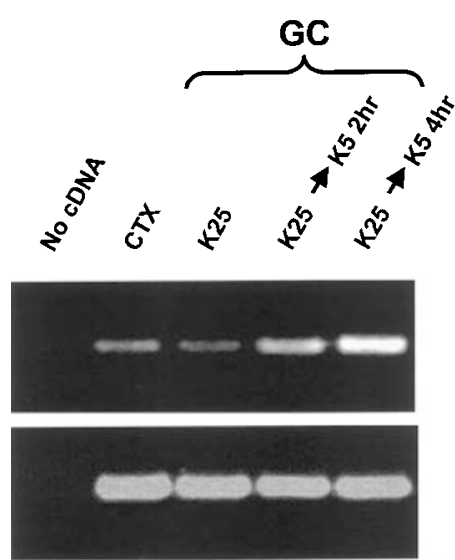

GD3 synthase (353 bp)

\section{$\beta$-actin (400 bp)}

Figure 5 RT - PCR analysis of GD3 synthase mRNA in cultured granule cells (GC) switched from K25 into sfK5 (2 and $4 \mathrm{~h})$. GD3 synthase in adult rat cerebral cortex (CTX) is also shown. $\beta$-Actin mRNA is shown as a control and to exclude contamination by genomic DNA (see Materials and Methods)

that the increase in GD3 formation was causally related to apoptosis we used two strategies: (i) the application of exogenous GD3 to cultures; and (ii) the depletion of endogenous GD3 by using GD3 synthase antisenses. Exogenous GD3 had no effect on the viability of K25 cultures, perhaps because the trophic input provided by chronic depolarization is strong enough to overcome an apoptotic signal. However, GD3 accelerated the development of 'low$\mathrm{K}^{+}$-induced apoptosis and, remarkably, almost $20 \%$ of granule cells treated with GD3 acquired the apoptotic phenotype as early as $4 \mathrm{~h}$ after the medium switch. Such a rapid development of apoptosis strongly supports a role for GD3 in neuronal death. GD3 synthase antisenses applied to cultures switched from $\mathrm{K} 25$ into scK10 reduced the extent of apoptosis by about $40-50 \%$, although it abolished early increase in GD3 levels. This suggests that an enhanced formation of GD3 contributes to, but is not the only factor involved in the induction of neuronal apoptosis. Other factors might become predominant under more severe deathinducing conditions, as observed in cultures switched from K25 into sfK5. The lack of protection by GD3 synthase antisenses inthe latter condition is consistent with this view.

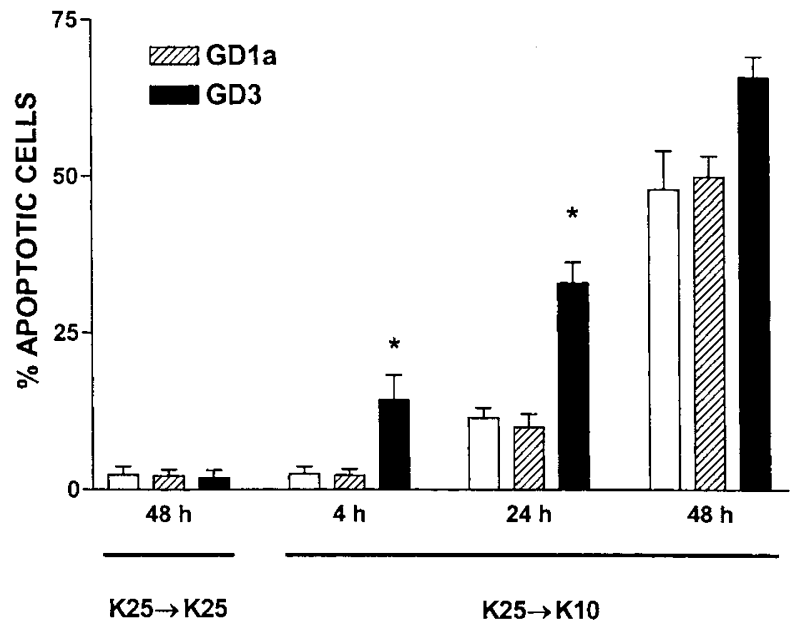

Figure 6 Effect of exogenous application of GD3 or GD1a (both at $10 \mu \mathrm{M}$ ) on the percentage of apoptotic neurons in cultures switched from a K25 medium into a scK10 medium collected from sister cultures. In this particular experiment, the percentage of apoptotic neurons was determined by FACS analysis after DNA staining with propidium iodide. Values are means \pm S.E.M. of 3-9 determinations. ${ }^{*} P<0.05$ (One-way ANOVA+Fisher's PLSD) vs values obtained from cultures switched from K25 into K10 and treated with buffer or GD1a

Perhaps GD3 is mandatory or dispensable depending on how much additional factors partecipate to the activation of the mitochondrial pathway of cell death. Thus, only if the intensity of the apoptogenic stimulus is mild, GD3 can contribute to reach the threshold for the activation of the mitochondrial cascade leading to Cytochrome $c$ release and caspase activation. However, we cannot exclude that neuroprotection deriving from the knock-down of GD3 synthase in cultures switched from K25 into scK10 depends on an increased formation of gangliosides of the 'a' series from the common precursor, GM3. Accordingly, the gangliosides GM1 and GD1a or their semisynthetic derivatives are protective against apoptosis induced by ethanol- or 'low- $\mathrm{K}^{+}$' in cultured cerebellar granule cells, ${ }^{29,30}$ or by serum deprivation, ionomycin or cyclosporin A in cultured cortical neurons. ${ }^{31}$

In conclusion, present results provide the first evidence that an early and transient increase in the GD3 ganglioside 


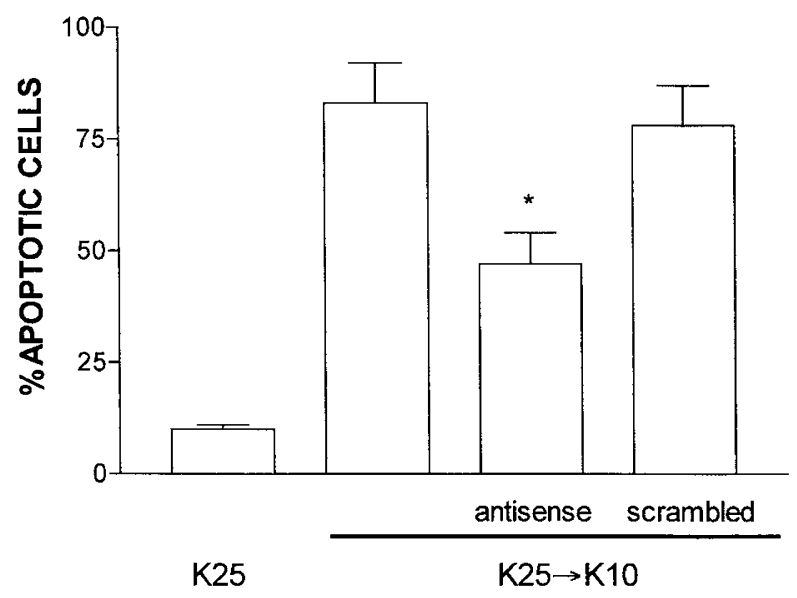

Figure 7 Antisense oligonucleotides directed against GD3 synthase reduced the extent of apoptotic death in cultured granule cells. Cultures were switched from a K25 medium into a scK10 medium collected from sister cultures. Antisenses or scrambled oligonucleotides (both at $2 \mu \mathrm{M}$ ) were applied once a day for the 2 days preceding the medium switch, and then re-applied immediately after the switch. Apoptotic death was assessed by Hoechst staining. Values are means \pm S.E.M. of determinations from six culture dishes. ${ }^{*} P<0.05$ (One-way ANOVA+Fisher's PLSD) vs values obtained from cultures switched from K25 into scK10 that did not receive the antisense

is associated with, and causally related to, the development of apoptosis in cultured neurons. The mechanism(s) underlying the increased formation of GD3 and the metabolic source for GD3 synthesis are still obscure. An increased formation of ceramide through the acidic sphingomyelinase pathway is considered as a major source for cytotoxic GD3 in blood cells following Fas/CD95 receptor activation. ${ }^{4-7}$ However, there is no evidence that ceramide is produced from sphingomyelin in granule cells committed to death, although granule cells develop apoptosis in response to the cell permeable ceramide analogue, C2-ceramide. ${ }^{32-34}$ An induction of the Fas/CD95 ligand is consistently observed in granule cells switched into a low- $\mathrm{K}^{+}$-containing medium, ${ }^{35-37}$ but whether or not Fas/CD95 ligand contributes to the development of granule cell apoptosis is matter of controversy. ${ }^{36,37}$ Clearly, more studies are needed for the identification of the pathway leading to GD3 synthesis in neuronal apoptosis.

\section{Materials and Methods}

\section{Preparation of cultured granule cells}

Primary cultures of cerebellar granule cells were prepared from 8-day old Sprague-Dawley rats (Charles River, Calco, Italy) rats, as described previously. ${ }^{38}$ Cells were plated on $35-\mathrm{mm}$ dishes $\left(2 \times 10^{6} /\right.$ dish) in $2 \mathrm{ml}$ of basal Eagle's medium (Gibco) containing 10\% fetal calf serum and 25 or $10 \mathrm{mM} \mathrm{K}^{+}$as $\mathrm{KCl}$ (K25 and K10). Cytosine-Darabinofuranoside $(10 \mu \mathrm{M})$ was added $16-18 \mathrm{~h}$ after plating to avoid the proliferation of non-neuronal cells. Mature cultures contained $>90 \%$ of granule cells, $3-5 \%$ of GABAergic neurons and a small percentage of non-neuronal cells (mostly glial cells) as contami- nants. ${ }^{38}$ Cultures at 6-7 days in vitro (DIV) were used for all experiments. The culture medium was replaced with: (a) a fresh medium containing $5 \mathrm{mM} \mathrm{K}^{+}(\mathrm{K} 5)$ and lacking fetal calf serum; or (b) the medium collected from sister cultures grown in K10 containing serum since the time of plating. In control cultures, the medium was replaced with an identical medium from sister cultures grown in K25.

\section{Assessment of apoptotic death and of changes in mitochondrial membrane potential}

Apoptotic death was assessed by fluorescent chromatin staining with Hoechst 33258 , as described previously. ${ }^{16}$ Apoptotic cells were considered as those showing chromatin fragmentation or nuclear picnosis. The percentage of apoptotic neurones was also determined by FACS analysis after DNA staining with propidium iodide. Cultures were washed in PBS (pH 7.4), harvested with a cell scraper, and washed twice in PBS by low-speed centrifugation. The cell pellet was incubated for $1 \mathrm{~h}$ at $4^{\circ} \mathrm{C}$ in $70 \%$ ethanol, washed again in PBS, and finally stained in the dark for $1 \mathrm{~h}$ with $100 \mu \mathrm{g} / \mathrm{ml}$ of propidium iodide in PBS. DNA content and ploidy was assessed by using an Epics Coulter $\mathrm{XL}$ flow cytometer. To assess whether granule cell apoptosis was associated with changes in mitochondrial membrane potentials, we have incubated the cultures with the fluorescent dye $5,5^{\prime}, 6,6^{\prime}$ tetrachloro-1,1',3,3'tetraethylbenzimidazolocarbocyanine iodide (JC1 ), which is sensitive to changes in mitochondrial membrane potential. ${ }^{39}$ Cultures were incubated with $3 \mu \mathrm{M} \mathrm{JC}-1$ for $20 \mathrm{~min}$ at $37^{\circ} \mathrm{C}$ and then washed in PBS. Green and red fluorescence was detected by a Zeiss laser scanner microscope (LSM510, Oberkochen, Germany).

\section{Assessment of intracellular GD3 levels}

Cultures were washed twice with ice-cold PBS $(\mathrm{pH} 7.4)$ and cells were scraped from the dishes and homogenized. Gangliosides were extracted according to the method of Svennerholm and Fredman ${ }^{40}$ as described previously ${ }^{41}$ and analysed by high performance thin layer chromatography (HPTLC) using analysitical pre-coated Silica gel 60 HPTLC plates (Merck, Darmstadt, Germany). All plates were first activated by heating to $100^{\circ} \mathrm{C}$ for $30 \mathrm{~min}$. Samples were spotted onto plates with a Hamilton syringe in chrloroform-methanol- $0.25 \% \mathrm{KCl}$ $(5: 4: 1, \mathrm{vol} / \mathrm{vol} / \mathrm{vol})$. When detection of all gangliosides was required, plates were air-dried and stained with resorcinol spray reagent, which stains sialic acid-containing glycolipids. Gangliosides GM3, GM1, GD1a, GD1b and GT1b (Sigma, St. Louis, MO, USA), GM2 and GD3 (kindly provided from Fidia Research Laboratories, Abano Terme, Italy) were used as standards. In most of the experiments, we specifically detected the GD3 ganglioside using the R24 anti-GD3 monoclonal antibody $(1: 100)$. The plates were incubated for $1 \mathrm{~h}$ at room temperature with the primary antibody, washed twice with PBSTween-20, and then incubated for $45 \mathrm{~min}$ at room temperature with an horseradish peroxydase-conjugated rabbit anti-mouse antibody (1:200; Sigma). Detection was carried out by ECL (Amersham, Milan, Italy). The bands were quantified by scanning densitometric analysis (GS300, Hoefer Scientific Instruments, San Francisco, CA, USA).

\section{Immunofluorescence analysis of GD3}

Cultured granule cells were fixed with $2 \%$ paraformaldehyde. Following incubation with $3 \%$ non immunized mouse serum in PBS, the R24 anti-GD3 monoclonal antibody (1:100) was applied at $4{ }^{\circ} \mathrm{C}$ for $72 \mathrm{~h}$. Cells were washed three times and FITC-conjugated anti-mouse immunoglobulin (Cappel, 1:200) was applied for $1 \mathrm{~h}$ at room 
temperature to visualize the labelled sites. Nuclei were stained with propidium iodine $(50 \mu \mathrm{g} / \mathrm{ml})$ in PBS. Fluorescence was detected by a Zeiss laser scanner microscope (LSM510, Oberkochen, Germany).

\section{RT - PCR analysis of GD3 synthase}

Total RNA was extracted from the cultures as described by Auffray and Rougenon, ${ }^{42}$ except that cells were washed $2 \times$ with ice cold PBS and then scraped in $2 \mathrm{ml}$ of cold $3 \mathrm{M} \mathrm{LiCl} / 6 \mathrm{M}$ Urea and the procedure was scaled down appropriately. Total RNA was finally subjected to Dnasel treatment (Boheringer Mannheim) according to manufacturer's instructions. Two $\mu \mathrm{g}$ of total RNA were then employed for cDNA synthesis, using Superscript II (BRL Life Tech.) and an oligodT primer according to manufacturer's instructions. The RT product was diluted to $100 \mu \mathrm{l}$ with sterile, distilled water and $1 \mu \mathrm{l}$ of CDNA was employed in each subsequent PCR amplification. Amplification of GD3 synthase cDNA was carried out employing the following primers: forward (5'CCAGCATAATTCGCCAGAGA-3 $\left.{ }^{\prime}\right)$ and reverse $\left(5^{\prime}\right.$-TTGCATGTTCACGGAGAAGG-3'). For $\beta$-actin cDNA amplification, the primers were those described by Roelen et al., ${ }^{43}$ which span an intron and yield products of different sizes depending on whether cDNA or genomic DNA is employed as a template (400 bp for a cDNA-derived product and $600 \mathrm{bp}$ for a genomic DNA-derived amplification). Reaction conditions included an initial denaturation step $\left(94^{\circ} \mathrm{C} / 3\right.$ min) followed by 45 cycles of $\left(94^{\circ} \mathrm{C} / 30 \mathrm{~s} ; 55^{\circ} \mathrm{C} / 30 \mathrm{~s} ; 72^{\circ} \mathrm{C} / 30 \mathrm{~s}\right)$. A final extension step $\left(72^{\circ} \mathrm{C} / 10 \mathrm{~min}\right)$ concluded the reaction. PCR products ( $1 / 3$ of the reaction) were analyzed electrophoretically on $2 \%$ agarose gels poured and run in $1 \times \mathrm{TAE}$.

\section{Addition of gangliosides or antisense oligonucleotides to the cultures}

GD3 and GD1a were dissolved in methanol and then dried under nitrogen. The two gangliosides were then suspended in water and applied to the cultures. Cultures were also treated with the following 'end-capped' phosphorothioate antisense oligonucleotide directed against the enzyme $\alpha$-2,8-sialyltransferase (GD3 synthase): $5^{\prime}-\mathrm{CAG}$ TAC AGC CAT GGC CCC TCT-3'. A scrambled oligonucleotide was used as a control: 5'-CGA CCT ACC TAT GCG CTA CCG-3'. Oligonucleotides were applied to cultures at 4-5 DIV once per day for 2 days. Afterwards, the culture medium was replaced with a medium collected from sister cultures grown in $\mathrm{K} 25$ or $\mathrm{K} 10$. For the detection of GD3, cultures were stopped $4 \mathrm{~h}$ later without any further addition of oligonucleotides. For the assessment of apoptotic death, oligonucleotides were applied once more immediately after the medium switch, and cultures were examined $48 \mathrm{~h}$ later.

\section{References}

1. Behl C (2000) Apoptosis in Alzheimer's disease. J. Neurol. Transm. 107: 13251344

2. Graham SH and Chen J (2001) Programmed cell death in cerebral ischemia. J. Cereb. Blood Flow Metab. 21: 99-109

3. Gibson RM (2001) Does apoptosis have a role in neurodegeneration? BMJ 322 : $1539-1540$

4. De Maria R, Lenti L, Malisan F, d'Agostino F, Tomassini B, Zeuner A, Rippo MR and Testi R (1997) Requirement for GD3 gaglioside in CD95- and ceramideinduced apoptosis. Science 277: 1652-1655

5. De Maria R, Rippo MR, Schuchman EH and Testi R (1998) Acidic sphingomyelinase (ASM) is necessary for fas-induced GD3 ganglioside accumulation and efficient apoptosis in lymphoid cells. J. Exp. Med. 187: $897-902$
6. Rippo MR, Malisan F, Ravagnan L, Tomassini B, Condol, Costantini P, Susin SA, Rufini A, Todaro M, Kroemer G and Testi R (2000) GD3 ganglioside directly targets mitochondria in a bcl-2-controlled fashion. FASEB J. 14: 2047-2054

7. Malisan F and Testi R (1999) Lipid signaling in CD95-mediated apoptosis. FEBS Lett 452: $100-103$

8. Kristal BS and Brown AM (1999) Apoptogenic GD3 directly induces the mitochondrial permeability transition. J. Biol. Chem. 274: 23169-23175

9. Scorrano L, Petronilli V, Di Lisa F and Bernardi P (1999) Commitment to apoptosis by GD3 ganglioside depends on opening of the mitochondrial permeability transition pore. J. Biol. Chem. 274: 22582-2585

10. Colell A, Garcia-Ruiz C, Roman J, Ballesta A and Fernandez-Checa JC (2001) Ganglioside GD3 enhances apoptosis by suppressing the nuclear factor-kappa B-dependent survival pathway. FASEB J. 15: 1068-1070

11. Gallo V, Kingsbury A, Balazs R and Jorgensen OS (1987) The role of depolarization in the survival and differentiation of cerebellar granule cells in culture. J. Neurosci. 7: 2203-2213

12. Balazs R, Jorgensen OS and Hack N (1988) N-Methyl-D-aspartate receptor promotes the survival of cerebellar granule cells in culture. Neurosci. 27: 437 451

13. D'Mello SR, Galli C, Ciotti T and Calissano P (1993) Induction of apoptosis in cerebellar granule cells by low potassium: inhibition of death by insulin-like growth factor-I and CAMP. Proc. Natl. Acad. Sci. USA 90: 10989-10993

14. D'Mello SR, Borodezt K and Soltoff SP (1997) Insulin-like growth factor and potassium depolarization maintain neuronal survival by distinct pathways: possible involvement of PI-3-kinase in IGF-I signalling. J. Neurosci. 17: 15481560

15. Copani A, Bruno VMG, Barresi V, Battaglia G, Condorelli DF and Nicoletti F (1995) Activation of metabotropic glutamate receptors prevents neuronal apoptosis in culture. J. Neurochem. 64: 101-108

16. Catania MV, Copani A, Calogero A, Ragonese GI, Condorelli DF and Nicoletti F (1999) An enhanced expression of the immediate early gene, Egr-1, is associated with neuronal apoptosis in culture. Neuroscience 91: 1529-1538

17. Schramm M, Eimerl S and Costa E (1990) Serum and depolarising agents cause acute neurotoxicity in cultured cerebellar granule cells: role of glutamate receptors responsive to $\mathrm{N}$-methyl-D-aspartate. Proc. Natl. Acad. Sci. USA 87: $1193-1197$

18. Svennerholm L, Rynmark BM, Vilbergsson G, Fredman P, Gottfries J, Mansson JE and Percy A (1991) Gangliosides in human fetal brain. J. Neurochem. 56: $1763-1768$

19. Percy AK, Gottfries J, Vilbergsson G, Mansson JE and Svennerholm L (1991) Glycosphingolipid glycosyltransferases in human fetal brain. J. Neurochem. 56: $1461-1465$

20. Goldman and Reynolds (1996) A reappraisal of ganglioside GD3 expression in the CNS. Glia 16: 291-295.

21. Kawai H, Sango K, Mullin KA and Proia RL (1998) Embryonic stem cells with a disrupted GD3 synthase gene undergo neuronal differentiation in the absence of b-series gangliosides. J. Biol. Chem. 273: 19634-19638

22. Kalanj S, Kracun I, Rosner H and Cosovic C (1991) Regional distribution of brain gangliosides in Alzheimer's disease. Neurol. Croat. 40: 269-281

23. Ando S, Toyoda $Y$, Nagai $Y$ and lkuta $F$ (1984) Alterations in brain gangliosides and other lipids of patients with Creutzfeld-Jakob disease and subacute sclerosing panencephalitis (SSPE). Jpn. J. Exp. Med. 54: 229-234

24. Fukumoto S, Mutoh T, Hasegawa T, Miyazaki H, Okada M, Goto J, Furukawa K and Urano T. GD3 synthase gene expression in PC12 cells results in the continuous activation of TrkA and ERK1/2 and enhanced proliferation. J. Biol. Chem. 275: $5832-5838$

25. Birkle S, Gao L, Zeng G and Yu RK (2000) Down regulation of GD3 ganglioside and its $\mathrm{O}$-acetylated derivative by stable transfection with antisense vector against GD3-synthase gene expression in hamster melanoma cells: effects on cellular growth, melanogenesis, and dendricity. J. Neurochem. 75: 547-554

26. Tsuji S, Kojima N and Hitoshi S (1996) New evidence for the occurrence of a glycolipid-mediated signal transduction system. J. Lipid. Mediat. Cell Signal 14: $289-294$

27. Cummer W and Zhang $\mathrm{H}$ (1996) Ganglioside GD3 in radial glia and astrocytes in situ in brain of young and adult mice. J. Neurosci. Res. 46: 18-23

28. Kawashima I, Nagata I and Tai T (1996) Immunocytochemical analysis of gangliosides in rat primary cerebellar cultures using specific monoclonal antibodies. Brain Res. 732: 75-86 
29. Saito M, Guidotti A, Berg MJ and Marks N (1998) The semisynthetic ganglioside LIGA20 potently protects neurons against apoptosis. Ann. NY Acad. Sci. 845: 253-262

30. Saito M, Berg MJ, Guidotti A and Marks N (1999) Gangliosides attenuate ethanolinduced apoptosis in ratcerebellargranule neurons. Neurochem Res. 24:11071115

31. Ryu BR, Choi DW, Hartley DM, Costa E, Jou l and Gwag BJ (1999) Attenuation of cortical neuronal apoptosis by gangliosides. J. Pharmacol. Exp. Ther. 290:811 816

32. Monti B, Zanghellini P and Contestabile A (2001) Characterization of ceramideinduced apoptotic death in cerebellar granule cells in culture. Neurochem. Int. 39: $11-18$

33. Tanikawi T, Yamada T, Alahara H, Ohyagi Y and Kira J (1999) Ceramide induces apoptosis to immature cerebellar granule cells in culture. Neurochem Res. 24: $685-690$

34. Centeno F, Mora A, Fuentes JM, Soler G and Claro E (1998) Partial lithiumassociated protection against apoptosis induced by $\mathrm{C} 2$-ceramide in cerebellar granule neurons. Neuroreport 9: 4199-4203

35. Ginham R, Harrison DC, FacciL, Skaper Sand PhilpottKL (2001) Upregulation of death pathway molecules in rat cerebellar granule neurons undergoing apoptosis. Neurosci Lett. 302: 113-116

36. Le-Niculescu H, Bonfoco E, Kasuya Y, Claret FX, Green DR and Kari M (1999) Withdrawal of survival factors results in the activation of the JNK pathway in neuronal cells leading to fas ligand induction and cell death. Mol. Cell Biol. 19: $751-763$
37. Gerhardt E, Kugler S, Leist M, Beier C, Berliocchi L, Volbracht C, Weller M, Bah M, Nicotera P and Schulz JB (2001) Cascade of caspase activation in potassiumdeprived cerebellar ranule neurons: targets for treatment with peptide and protein inhibitors of apoptosis. Mol. Cell Neurosci. 17: 717-731

38. Nicoletti F, Wroblewski JT, Novelli A, Alho H, Guidotti A and Costa E (1986) The activation of inositol phospholipid hydrolysis as a signal transducing system for excitatory amino acids in primary cultures of cerebellargranule cells. J. Neurosci. 6: $1905-1911$

39. Cossarizza A, Baccarani-Contri M, Kalashnikova G and Franceschi C (1993) A new method for the cytofluorimetric analysis of mitochindrial membrane potential using the J-aggregate forming lipophilic cation 5,5',6,6'-tetrachloro-1-1',3,3'tetraethylbenzimidazolcarbocyanine iodide (JC-1). Biochem. Biophys. Res Commun. 197: 40-45

40. Svennerholm $L$ and Fredman $P$ (1980) A procedure for the quantitative isolation of brain gangliosides. Biochem. Biophys. Acta. 617: 97-109

41. Dotta F, Previti M, Neerman-Arbez M, Dionisi S, Cucinotta D, Lenti L, Di Mario U and Halban PA (1998) The GM2-1 ganglioside islet autoantigen in insulindependent diabetes mellitus is expressed in secretory granules and is not $\beta$-cell specific. Endocrinology 139: 316-319

42. Auffrey $C$ and Rougeon $F$ (1980) Purification of mouse immunoglobulin heavy chain messenger RNAs from total myeloma tumor RNA. Eur. J. Biochem. 107: 303-314

43. Roelen BA, Lin HY, Knezevic V, FreundE and Mummery CL (1994) Expression of TGF-beta and their receptors during implantation and organogenesis of the mouse embryo. Dev. Biol. 166: 716-728 